\title{
Cellular oxygen sensing and the anesthesiologist: the Nobel- worthy discovery of hypoxia inducible factor and its implications in clinical practice
}

\author{
Gregory M. T. Hare, MD, PhD
}

Received: 25 October 2019/Revised: 28 October 2019/Accepted: 29 October 2019/Published online: 11 November 2019

(C) Canadian Anesthesiologists' Society 2019

On October 7 2019, the Nobel committee awarded the prize in physiology or medicine to Drs Gregg Semenza (Johns Hopkins University), Peter Radcliffe (Oxford University), and William Kaelin Jr. (Harvard University) for their discovery of hypoxia inducible factor (HIF) and associated mechanisms of oxygen sensing and biological regulation. ${ }^{1,2}$ The committee noted that the prize was awarded for "establishing the basis of our understanding of how oxygen levels affect cellular metabolism and physiological function". ${ }^{1}$ The importance of their discovery has been long understood by many across both basic science and medical fields, and was acknowledged by the Canadian Gairdner Institute in 2010. ${ }^{3}$

In many senses, this is also a definition of one of the central roles of practicing anesthesiologists-i.e., to preserve optimal perfusion and oxygen delivery to the body's tissues.

\section{The history of oxygen discovery and its importance to physiology}

The current level of oxygen in the atmosphere has evolved over the past billion years allowing complex (metazoan) organisms including humans to develop. ${ }^{4}$ From the earliest days, populations have been able to adapt to life at lower oxygen levels at altitude as exemplified by the Himalayan and Andean cultures. From a Western scientific perspective, the collaborative research of Carl Wilhelm Scheele (1742-1786), who first published on the nature of oxygen; Antoine Lavoisier (1743-1794), who coined the term "oxygen" as the acidic portion of the atmosphere that

G. M. T. Hare, MD, PhD ( $\varangle)$

Department of Anesthesia, St. Michael's Hospital, University of Toronto, 30 Bond Street, Toronto, ON M5B 1W8, Canada

e-mail: greg.hare@unityhealth.to supported combustion; and Joseph Priestly (1733-1804), who most clearly showed evidence that oxygen production by plants was necessary for animal survival, we have continued to focus on the importance of oxygen to support our survival. ${ }^{5}$ Of interest, one of the scientific discoveries linking hypoxia at altitude with changes in hemoglobin $(\mathrm{Hb})$, initiated via HIF-dependent erythropoietin (EPO) expression, was achieved by Mabel Purefoy FitzGerald (honorary Master of Arts, Oxford University). As a member of Haldane's Pike's Peak Expedition, she independently provided one of the first observations of the relationship between reduced partial pressure of oxygen exposure at increased altitude and increased $\mathrm{Hb}$ concentration in men and women. ${ }^{6}$

\section{The discovery of HIF}

The discovery of HIF and its biological significance was derived from collaborative research performed over many years. The known relationship between EPO expression and hypoxia led Gregg Semenza to ask "what is the element that promotes EPO transcription under hypoxic conditions?" Ingenious investigation at the molecular level identified a "cis" element that acted at a location on the DNA ahead of the EPO gene to turn the gene on and increase gene transcription. This line of investigation led to the discovery of HIF-1, the nuclear binding factor that promoted EPO expression in isolated cells. ${ }^{8}$ Working in parallel, Peter Ratcliffe explored the molecular and physiologic regulation of EPO transcription and its biological expression. He determined the location of the EPO gene transcription promoter ${ }^{9}$ and defined the mechanism of renal EPO expression and its secretion in response to graded degrees of anemia and hypoxia. ${ }^{10,11}$ Finally, approaching the problem from an additional angle, William Kaelin Jr. explored the role of von Hipple Lindau 
(VHL) protein and its impact on the expression of hypoxia inducible genes under conditions of changes in ambient oxygen. ${ }^{12}$ His research characterized the relationship between VHL and HIF by showing that binding of the two molecules only occurred if HIF was hydroxylated at its proline residues. Such hydroxylation enabled VHL binding and the subsequent destruction of the HIF-VHL protein complex via proteolytic degradation pathways in the presence of oxygen. ${ }^{13}$ In the absence of oxygen, such proline-hydroxylation does not occur, thus preventing VHL binding and VHL-HIF degradation resulting in the stabilization of HIF. ${ }^{14}$ Together, the discoveries made by these three researchers (and their many collaborators) led the way to a profound new understanding of the physiologic and cellular adaptation to hypoxia and cellular capacity for oxygen sensing. By virtue of this mechanism, every cell in the mammalian body has the capacity for oxygen sensing. This capacity has provided the required mechanism to drive integrative physiologic adaptations that occur in response to hypoxemia, acute blood loss, hemorrhage, and anemia frequently observed in our acute care patients.

\section{Discovery through collaboration}

In a world of ever-increasing competition, with an overemphasis on becoming "independent", the research programs that evolved from the mutual discoveries of these three leaders can only be described as truly collaborative. Indeed, one member of the Nobel committee described the co-dependent interaction between these investigators as a "three-legged stool", emphasizing that each leg was essential to achieve the discovery. ${ }^{2}$ In reference to the value of this collaboration and discovery, William Kaelin Jr. eloquently summarized the impact as follows: "Occasionally you understand something that has never been understood before, and if you are really lucky you might discover a mechanism that is so beautiful that it makes your jaw drop. That is the real prize - to be able to participate in that process". ${ }^{1}$

\section{How might this discovery impact our practice as anesthesiologists?}

Our understanding of HIF biology, and its role in regulating oxygen homeostasis, has incorporated an expanding number of biological systems and is known to involve over 4,000 genes. ${ }^{1}$ As our understanding continues to evolve, the potential for therapeutic intervention grows. One example of how this biology may help improve the health and well-being of our patients may be the effective treatment of acute and chronic anemia. Anemia is a global health problem. Indeed, an estimated one third of the world's population suffers from anemia, which is a leading cause of disability in youth and young adults. ${ }^{15,16}$ Despite this known baseline risk, an estimated $30 \%$ of patients undergoing elective surgical procedures have been identified as being anemic. ${ }^{17}$ Furthermore, we know that even mild to moderate anemia is associated with an increased risk for perioperative morbidity and mortality in patients undergoing elective cardiac and non-cardiac surgery. ${ }^{17,18}$ Finally, acute hemodilution and blood loss, which frequently occur during surgery, are also associated with a further increased incidence of severe adverse outcomes. ${ }^{19}$

Thus, anemia is an identified risk to our patients. But, what can we do to improve patient outcomes? First, understanding anemia as a risk ${ }^{20}$ has supported the development of effective multidisciplinary blood management programs, such as the Ontario Network of Transfusion Coordinators (ONTraC), which have been shown to effectively treat anemia, minimize blood transfusion, and improve patient outcomes. ${ }^{21}$ Second, defining anemia as a risk has re-focused research on the importance of oxygen delivery to tissue, and its limitation, as a potential mechanism of injury. From human and animal studies, ${ }^{22,23}$ we know that the true conditions of tissue oxygen delivery cannot be successfully mimicked in the test tube, or fully defined by studies in cultured cells. Thus, accurate assessment of the impact of anemia on tissue hypoxia must be performed using integrative whole organism physiological approaches. Utilizing whole animal transgenic models derived from knowledge of the HIF discovery, ${ }^{24}$ we understand that anemia leads to multi-organ tissue hypoxia and enhanced HIF expression in tissues of anemic animals. ${ }^{23}$ Furthermore, interrupting HIF expression has been shown to increase mortality in animal models of acute anemia, ${ }^{23}$ indicating that HIF-dependent mechanisms support survival during anemia. Thirdly, clinical research has assessed whether measuring levels of hypoxic serum biomarkers, such as EPO, in the blood can predict the risk of tissue hypoxia and organ injury (e.g., the kidney). ${ }^{25}$ This approach may help to identify anemic patients at risk of acute organ injury and direct effective treatments, including treatment of preoperative anemia and targeted blood transfusion, in patients at risk who experience acute surgical blood loss.

Understanding the biology of HIF-dependent transcription of EPO, and the role of proline-hydroxylation in the degradation of HIF has led to the development and assessment $^{24}$ of novel peptides that can inhibit prolylhydroxylase enzymes responsible for HIF degradation, thereby stabilizing HIF and increasing the expression of HIF-dependent molecules such as EPO. ${ }^{26}$ This approach may directly promote erythrogenesis as a primary goal to treat anemia. Completed phase-III clinical trials have shown the efficacy of these novel oral peptide drugs with respect to 
treating anemia and improving Hb levels. ${ }^{26}$ This new class of drugs may both treat anemia and enhance hypoxic cellular defense mechanisms to enhance tolerance of anemia and improve patient outcomes. Nevertheless, close examination for potential adverse effects of generalized enhancement of HIF-dependent gene expression must also be maintained.

In summary, the discovery of HIF and its multiple integrated physiologic mechanisms is of great value to our understanding of human biology. It lends to a greater understanding of the importance of how our complex biological systems regulate and utilize oxygen under times of plenty and times of need. Ongoing and future studies will be required to determine whether or not, therapeutic interventions based on this knowledge will improve patient outcomes and quality of life. As anesthesiologists, we remain focused on maintaining optimal oxygen delivery to vital organs through an understanding of the integrated physiologic function of all organ systems in the acute care of patients who are frequently exposed to the risk of hypoxia and acute blood loss. As such, we are well-positioned to contribute to the ongoing application of new treatments to optimize patients' outcomes, in part, based on our understanding of the risk of tissue hypoxia and the role of HIF biology in ensuring adequate oxygen delivery to tissues.

\section{Conflicts of interest None.}

Funding statement Merit Award, Department of Anesthesia, University of Toronto.

Editorial responsibility This submission was handled by Dr. Hilary P. Grocott, Editor-in-Chief, Canadian Journal of Anesthesia.

\section{References}

1. Burki T. 2019 Nobel Prize awarded for work on oxygen regulation. Lancet 2019; 394: 1399-400.

2. Ledford H, Callaway E. Biologists who decoded how cells sense oxygen win medicine Nobel. Nature 2019; 574: 161-2.

3. Webster PC. The winners of the. Gairdner Awards. Lancet 2010; DOI: https://doi.org/10.1016/S0140-6736(10)60526-0.

4. Taylor CT, McElwain JC. Ancient atmospheres and the evolution of oxygen sensing via the hypoxia-inducible factor in metazoans. Physiology (Bethesda) 2010; 25: 272-9.

5. Severinghaus $J W$. Fire-air and dephlogistication. Revisionisms of oxygen's discovery. Adv Exp Med Biol 2003; 543: 7-19.

6. West JB. Centenary of the Anglo-American high-altitude expedition to Pikes Peak. Exp Pysiol 2012; 97: 1-9.

7. Semenza GL, Dureza RC, Traystman MD, Gearhart JD, Antonarakis SE. Human erythropoietin gene expression in transgenic mice: multiple transcription initiation sites and cisacting regulatory elements. Mol Cell Biol 1990; 10: 930-8.

8. Semenza GL, Nejfelt MK, Chi SM, Antonarakis SE. Hypoxiainducible nuclear factors bind to an enhancer element located 3' to the human erythropoietin gene. Proc Natl Acad Sci USA 1991; 88: 5680-4.
9. Pugh CW, Tan CC, Jones RW, Ratcliffe PJ. Functional analysis of an oxygen-regulated transcriptional enhancer lying 3' to the mouse erythropoietin gene. Proc Natl Acad Sci USA 1991; 88: 10553-7.

10. Eckardt KU, Koury ST, Tan CC, et al. Distribution of erythropoietin producing cells in rat kidneys during hypoxic hypoxia. Kidney Int 1993; 43: 815-23.

11. Tan CC, Eckardt KU, Firth JD, Ratcliffe PJ. Feedback modulation of renal and hepatic erythropoietin mRNA in response to graded anemia and hypoxia. Am J Physiol 1992; 263: F474-81.

12. Iliopoulos $O$, Levy AP, Jiang C, Kaelin WG Jr, Goldberg MA. Negative regulation of hypoxia-inducible genes by the von Hippel-Lindau protein. Proc Natl Acad Sci USA 1996; 93: 10595-9.

13. Kaelin $W G J r$, Ratcliffe PJ. Oxygen sensing by metazoans: the central role of the HIF hydroxylase pathway. Mol Cell 2008; 30: 393-402.

14. Min JH, Yang H, Ivan M, Gertler F, Kaelin WG Jr, Pavletich NP. Structure of an HIF-1alpha -pVHL complex: hydroxyproline recognition in signaling. Science 2002; 296: 1886-9.

15. Williams AM, Addo OY, Grosse SD, et al. Data needed to respond appropriately to anemia when it is a public health problem. Ann NY Acad Sci 2019; 1450: 268-80.

16. Kassebaum NJ, Jasrasaria R, Naghavi $M$, et al. A systematic analysis of global anemia burden from 1990 to 2010. Blood 2014; 123: 615-24.

17. Fowler AJ, Ahmad T, Abbott TE, et al. Association of preoperative anaemia with postoperative morbidity and mortality: an observational cohort study in low-, middle-, and high-income countries. Br J Anaesth 2018; 121: 1227-35.

18. Musallam KM, Tamim HM, Richards T, et al. Preoperative anaemia and postoperative outcomes in non-cardiac surgery: a retrospective cohort study. Lancet 2011; 378: 1396-407.

19. Padmanabhan H, Siau K, Curtis J, et al. Preoperative anemia and outcomes in cardiovascular surgery: systematic review and metaanalysis. Ann Thorac Surg 2019; DOI: https://doi.org/10.1016/j. athoracsur.2019.04.108.

20. Shander A, Javidroozi M, Ozawa S, Hare GM. What is really dangerous: anaemia or transfusion? Br J Anaesth 2011; 107(Suppl 1): i41-59.

21. Hare GM, Freedman J, Mazer CD. Review article: risks of anemia and related management strategies: can perioperative blood management improve patient safety? Can J Anesth 2013; 60: 168-75.

22. Balaban DY, Duffin J, Preiss D, et al. The in-vivo oxyhaemoglobin dissociation curve at sea level and high altitude. Respir Physiol Neurobiol 2013; 186: 45-52.

23. Tsui AK, Marsden PA, Mazer CD, et al. Priming of hypoxiainducible factor by neuronal nitric oxide synthase is essential for adaptive responses to severe anemia. Proc Natl Acad Sci USA 2011; 108: 17544-9.

24. Safran M, Kim WY, O'Connell F, et al. Mouse model for noninvasive imaging of HIF prolyl hydroxylase activity: assessment of an oral agent that stimulates erythropoietin production. Proc Natl Acad Sci USA 2006; 103: 105-10.

25. Hare GM, Han K, Leshchyshyn $Y$, et al. Potential biomarkers of tissue hypoxia during acute hemodilutional anemia in cardiac surgery: a prospective study to assess tissue hypoxia as a mechanism of organ injury. Can J Anesth 2018; 65: 901-13.

26. Chen N, Hao C, Peng X, et al. Roxadustat for anemia in patients with kidney disease not receiving dialysis. N Engl J Med 2019; 381: 1001-10.

Publisher's Note Springer Nature remains neutral with regard to jurisdictional claims in published maps and institutional affiliations. 